\title{
Mejora de la calidad del acero obtenido por colada continua mediante influencias electrofísicas $^{(\cdot)}$
}

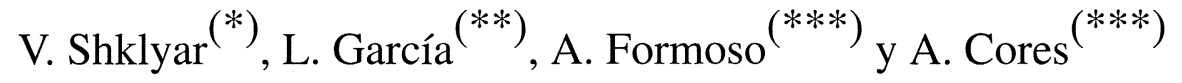 \\ Resumen Se han realizado ensayos de laboratorio con estaño fundido en crisol y a escala industrial con acero \\ líquido vertidos en moldes, sometiendo estos metales a influencias electrofísicas. Como resultado del \\ campo aplicado, en el estaño se observa que durante la solidificación varía la temperatura de cristali- \\ zación y el tiempo de solidificación. En el lingote de acero se observa un crecimiento de los cristales \\ en dirección del campo eléctrico y un aumento del límite de rotura. \\ Palabras clave: Conformación magnética. Colada electromagnética. Colada continua del acero.

\section{Improvement in steel quality obtained by continuous casting by means of electrophysical influences}

\begin{abstract}
Laboratory tests with tin smelted in a crucible and industrial tests with crude steel poured in moulds had been carried out. These metals were subjected to electrophysical influences. As a result of the applied electric field, it is observed in tin that during the solidification, the crystalization temperature and the solidification time are changed. Crystal growth in the electric field direction and an increase of the fracture limit in the steel ingot are observed.
\end{abstract}

Keywords: Magnetic shaping. Electromagnetic casting. Continuous casting of steel.

\section{INTRODUCCIÓN}

La mayoría de los defectos de superficie de un lingote se originan en la etapa inicial de solidificación en el molde. Con objeto de evitar la falta de uniformidad durante el enfriamiento en el molde y las fluctuaciones de los meniscos que actúan sobre la capa solidificada en la etapa inicial, se han realizado investigaciones sobre la colada electromagnética, especialmente aplicadas en el campo del aluminio (1 y 2). Esta tecnología aún no ha tenido un

(•) Trabajo recibido el día 27 de agosto de 1997 y aceptado en su forma final el 29 de octubre de 1998.

(*) Universidad Estatal Tecnológica de Donetsk. Av. Arteoma, 58. 34006-Donetsk (Ucrania).

(**) Unión de Empresas de Recuperación de Materias Primas. 1ra. Avda. 1604 Miramar. 11300 Ciudad de La Habana (Cuba).

(***) Centro Nacional de Investigaciones Metalúrgicas, CENIM (CSIC). Av. de Gregorio del Amo, 8. 28040Madrid (España). desarrollo completo en el campo del acero, a causa de la conductividad eléctrica más baja y densidad más elevada del acero comparado con el aluminio fundido.

Por otra parte, se han realizado investigaciones con el objetivo de aplicar la colada electromagnética a la colada continua del acero (3-5). Los resultados obtenidos señalan que los beneficios que se consiguen con la utilización de esta tecnología son:

- disminución de la presión ferroestática en el molde,

- mejora de la lubricación entre el material fundido y el molde, y

- mejora de la forma y de las fluctuaciones del menisco.

En el presente trabajo se estudia el comportamiento de la colada continua del acero bajo la influencia de un campo eléctrico y la mejora que se consigue con este tratamiento. 


\section{FUNDAMENTOS TEÓRICOS}

En los últimos años, cada vez se emplean más las influencias electrofísicas durante la cristalización del metal obtenido por colada continua (6 y 7). Sin embargo, este mecanismo de influencia no está completamente esclarecido, ya que la electrodinámica parte del supuesto de que al interior de un conductor ideal no penetra un campo electrostático externo. Para un conductor heterogéneo, que tiene un gradiente de temperatura, que en este caso específico es el metal que se cristaliza, esto no es así. En los conductores heterogéneos las cargas eléctricas se redistribuyen en su interior. Este comportamiento es función del campo eléctrico creado por la presencia interna de cargas eléctricas, expresada por la ecuación:

$$
\rho=-\varepsilon_{0} \cdot \vec{E}(\nabla \sigma / \sigma)
$$

donde

$\rho=$ densidad de carga $\mathrm{kl} / \mathrm{m}^{3}$

$\varepsilon_{0}=$ constante dieléctrica $\left(\varepsilon_{0}=8,85 \times 10^{-12}\right)$, $\mathrm{F} / \mathrm{m}$;

$\nabla=$ hamiltoniano;

$\vec{E}=\vec{E}(\vec{r}, \vec{\tau})=$ tensión del campo eléctrico, $\mathrm{V} / \mathrm{m}$;

$\sigma=\sigma(\vec{r}) \quad=$ Conductibilidad eléctrica del metal $(\omega \cdot \mathrm{m})^{-1}$

Una generalización de la ley de Ohm para un metal calentado no uniformemente, con distribución heterogénea, se obtiene por el método de Onzager:

$$
j=\sigma \cdot \vec{E}-\sigma \cdot \alpha \nabla T-\sum_{\mathrm{k}=1}^{\mathrm{p}} \sum_{\mathrm{i}=1}^{\mathrm{N}} L_{\mathrm{ki}} \nabla\left(\mu_{\mathrm{i}} / T\right)
$$

donde

$j=$ densidad de la corriente eléctrica, $\mathrm{A} / \mathrm{m}^{2}$.

$M_{\mathrm{ki}}, L_{\mathrm{ki}}, L_{\mathrm{kQ}}=$ coeficientes de Onzager.

$\mu_{\mathrm{i}}=$ potencial químico del componente $i, \mathrm{~J} / \mathrm{kg}$.

$T=$ Temperatura, $\mathrm{K}$.

$$
\begin{gathered}
\sigma=-\sum_{\mathrm{i}, \mathrm{k}=1}^{\mathrm{p}} M_{\mathrm{ki}} q_{\mathrm{i}} \\
\sigma \cdot \alpha=-T^{2} \sum_{\mathrm{k}=1}^{\mathrm{p}} L_{\mathrm{kQ}}
\end{gathered}
$$

De la ec. [2] resulta que en el medio del conductor eléctrico heterogéneo puede surgir la corriente eléctrica, incluso en ausencia de un campo eléctrico dentro del metal $\left(\vec{E}_{\text {int }}=0\right)$.

Si el metal analizado no está conectado en circuito cerrado, entonces en este sistema la corriente eléctrica está ausente $(\vec{j}=0)$ y la distribución de las cargas será estacionaria. En este caso:

$$
\vec{E}_{\text {int }}=\alpha \nabla T+(1 / \sigma) \sum_{\mathrm{k}=1}^{\mathrm{p}} \sum_{\mathrm{i}=1}^{\mathrm{N}} L_{\mathrm{ki}} \nabla\left(\mu_{\mathrm{i}} / T\right)
$$

Así, el campo eléctrico interno que aparece en el metal penetra en el medio que lo rodea y puede detectarse allí. La interacción del campo eléctrico externo con el interno puede llevar a la variación de este último y, por consiguiente, influir en el proceso que provoca la formación de este campo, es decir, en el proceso de trasmisión de calor y masa.

\section{ENSAYOS DE LABORATORIO}

Para esclarecer el efecto de la influencia electrofísica en el metal que cristaliza, se realizaron una serie de ensayos en el dispositivo experimental que cuyo esquema se ofrece en la figura 1. Una muestra pesada de estaño (punto de fusión $=232{ }^{\circ} \mathrm{C}$ ), se calienta en un crisol de porcelana hasta $260{ }^{\circ} \mathrm{C}$ y, posteriormente, la masa fundida se deja enfriar y la temperatura se mide durante su enfriamiento.

A la masa fundida se suministra un potencial comprendido entre 0 y $15 \mathrm{kV}$ desde la fuente de tensión eléctrica mediante un electrodo. Alrededor del crisol se coloca un segundo electrodo de hoja metálica, que se deriva a tierra. La medición desde cada potencial se realiza por triplicado en muestras nuevas de estaño de peso fijo y se promedian los resultados.

En la figura 2 se incluyen algunos de los termogramas de solidificación obtenidos para diferentes magnitudes del potencial aplicado.

En la figura 3 se representa la dependencia entre el tiempo de cristalización del metal y la magnitud del campo eléctrico externo. Para pequeños potenciales $(2 \mathrm{kV})$ ocurre una reducción del tiempo de cristalización del $10 \%$, y para un potencial de 30 $\mathrm{kV}$, el tiempo de solidificación se reduce en 7,5\%.

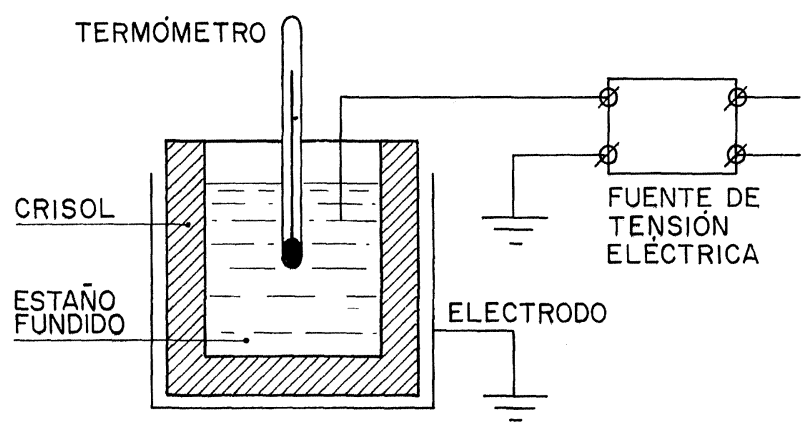

FIG. 1.-Estaño sometido a influencias electrofísicas.

FIG. 1.- Tin subjected to electrophysical influences. 


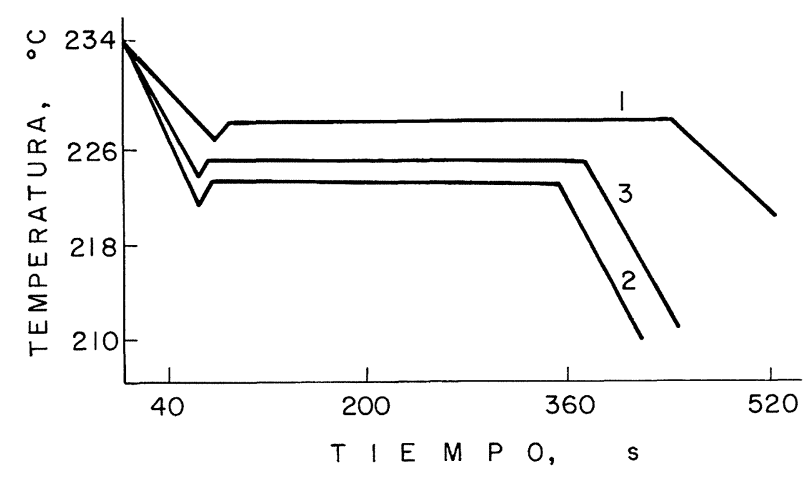

FIG. 2.- Termogramas de enfriamiento del estaño para $\Phi=0 \mathrm{kV}(1), 6 \mathrm{kV}(2)$ y $10 \mathrm{kV}(3)$.

FIG. 2.- Thermograms of tin coolling for $\Phi=0 \mathrm{kV}$ (1), $6 \mathrm{kV}(2)$ and $10 \mathrm{kV}(3)$.

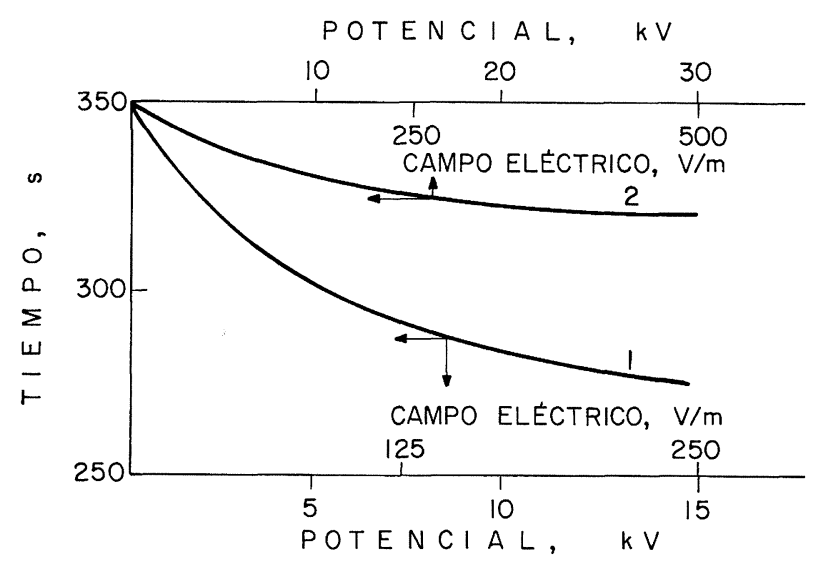

FIG. 3.- Dependencia entre el tiempo de cristalización del metal fundido y la magnitud del campo eléctrico externo. (1): $3 \mathrm{kV} \leq \Phi \leq 15 \mathrm{kV}$; (2): $0 \mathrm{kV} \leq \Phi \leq 30 \mathrm{kV}$.

Fig. 3.- Dependence of crystallization time of the melting metal and magnitude of external electric field. (1): $3 k V \leq \Phi \leq 15 k V$; (2): $0 k V \leq \Phi \leq 30 \mathrm{kV}$.

Por consiguiente, para reducir el tiempo de cristalización es efectivo utilizar pequeños potenciales.

Durante la solidificación del metal en presencia de un campo eléctrico disminuye la temperatura de equilibrio de la cristalización (Fig. 4). Para bajos potenciales de la fuente del campo eléctrico ésto no se observa, mientras que para altos potenciales, la temperatura de cristalización $T_{\mathrm{L}}$ disminuye entre 3$7{ }^{\circ} \mathrm{C}$. Este comportamiento puede estar relacionado con la disminución de calor en la transición de fase $L$, o con la variación del grado de ordenación del fundido que se recristaliza, que se caracteriza por variaciones de la entropía en la fase de transición:

$$
L=\Delta S \cdot T
$$

donde
$L=$ calor en la transición de fases, $\mathrm{kJ} / \mathrm{kg}$.

$\Delta S=$ variación de la entropía en la transición de fases, $\mathrm{kJ} / \mathrm{kg} \cdot \mathrm{K}$.

$T$ = temperatura, $\mathrm{K}$.

Winegard (8) demostró que la cristalización en presencia de un campo eléctrico es semejante a la cristalización bajo presión complementaria, y en este caso disminuye el calor (la temperatura) de la fase de transición. Esto varía las condiciones de solidificación. La velocidad de desplazamiento de la frontera de cristalización se determina por la transmisión de calor y de fases a través de una cubierta sólida (superficie solidificada). La disminución del calor, que se desprende durante la cristalización, acelera la solidificación de los lingotes.

En la estructura del metal, las propiedades mecánicas se determinan en gran parte por el ritmo instantáneo de cristalización:

$$
V_{\mathrm{cr}}=\partial P / \partial \tau
$$

donde

$V_{\mathrm{cr}}=$ velocidad de cristalización, $\mathrm{s}^{-1}$.

$P=$ porción de fase sólida en la masa fundida (varía entre 0 y 1 ).

$\tau=$ tiempo, $\mathrm{s}$.

El ritmo de cristalización en el estadio inicial depende en gran medida de la magnitud de subenfriamiento de la masa fundida. En presencia del campo eléctrico, la profundidad del subenfriamiento de la masa fundida se determina por la dirección e intensidad de este campo eléctrico (Fig. 4). En la figura, $T_{\mathrm{L}}$ es la temperatura de cristalización en la transición de fase en presencia de un campo eléctrico. Aquí, también, los pequeños campos eléctricos conducen a una notable disminución del subenfriamiento.

El subenfriamiento de la masa fundida es una "fuerza que se desplaza" de la cristalización. La variación de la magnitud del subenfriamiento de la masa fundida en el campo eléctrico externo corrobora la acción del campo en los cristales formados. Por tanto, para la formación de una nueva fase se requiere un subenfriamiento menor, entonces para estas mismas condiciones de trasmisión térmica externa se forman más cristales y a una mayor distancia de la superficie del lingote.

Este comportamiento se explica por la observación de una estructura del lingote de granos más finos (6). Las inclusiones no metálicas y los gases se desprenden en una zona de formación más amplia. Esto lleva a una distribución más uniforme de la segregación y al afino de las inclusiones no metálicas.

Por ello, la velocidad del crecimiento dendrítico también depende del subenfriamiento de la 


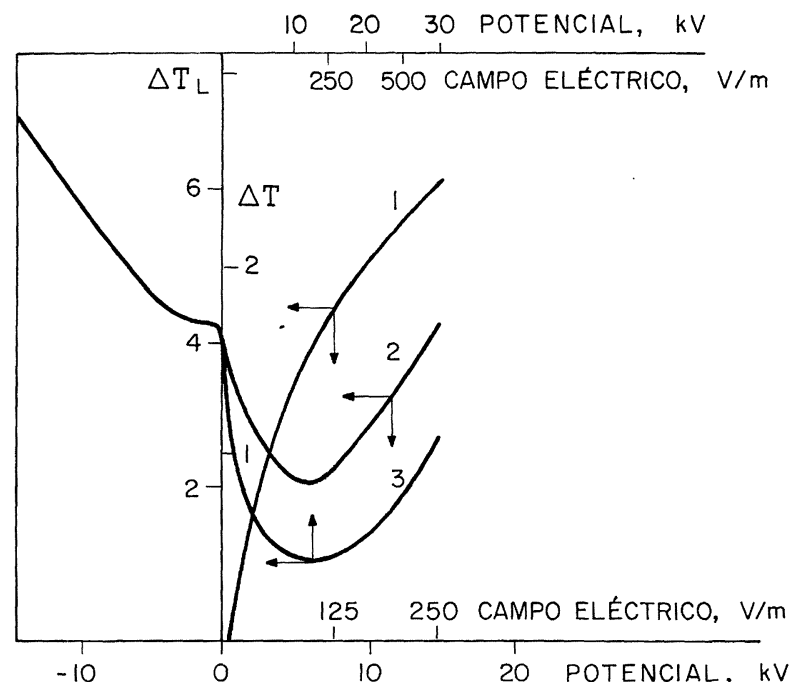

FIG. 4.- Variaciones de la temperatura de cristalización $\Delta T_{\mathrm{L}}=T_{\mathrm{L}}-T_{\mathrm{L}}^{\mathrm{E}}$, (1) y de subenfriamiento (2 y 3) del metal fundido bajo la acción del campo eléctrico externo.

FIG. 4.- Variations of crystallization temperature $\Delta \mathrm{T}_{\mathrm{L}}=\mathrm{T}_{\mathrm{L}}-\mathrm{T}_{\mathrm{L}}^{\mathrm{E}},(1)$ and undercooling $\left(\begin{array}{lll}2 & y & 3\end{array}\right)$ of the melting metal under the action of an external electrical field.

superficie del líquido ante los cristales que crecen; entonces, en presencia del campo eléctrico aumenta la velocidad del crecimiento dendrítico. Esto lleva consigo la variación del contenido de impurezas en la zona de fase doble, es decir el grosor de la propia zona de fase doble.

La disminución del ancho de la zona de fase doble, durante la cristalización del metal en campo eléctrico, permite su utilización efectiva en la industria de las aleaciones que tienden a la segregación.

Las dependencias obtenidas de la influencia del campo eléctrico externo sobre los parámetros de la cristalización permiten controlar las propiedades de los metales en las coladas industriales.

\section{ENSAYOS INDUSTRIALES}

Los ensayos se realizaron en la máquina de colada continua de palanquillas de la fábrica siderúrgica de Donetsk (Ucrania), con acero elaborado en horno eléctrico de arco con una composición en valores porcentuales de 0,53-0,58 C, 0,60-0,90 Mn, 1,50$2,00 \mathrm{Si}, \geq 0,30 \mathrm{Cr}, \geq 0,025 \mathrm{P}$ y $\leq 0,025 \mathrm{~S}$.

El acero líquido se vierte en moldes refrigerados con sección de $250 \times 500 \mathrm{~mm}$, colocando un campo eléctrico externo mediante un electrodo, al que se conduce una tensión constante de $12 \mathrm{kV}$. El segundo electrodo de la fuente hace tierra en el molde.

La corriente eléctrica, que pasa a través del acero colado, es de $80 \mathrm{~mA}$, por tanto la magnitud del calor en julios es despreciable. El acero se vierte con una velocidad de $0,5 \mathrm{~m} / \mathrm{min}$ a través de un vaso de circonio de $40 \mathrm{~mm}$ de diámetro a una temperatura de $1.490{ }^{\circ} \mathrm{C}$. Se realizan coladas de control (sin aplicación del campo eléctrico) y coladas experimentales. Se preparan probetas transversales y longitudinales del acero colado y con ellas se realizan investigaciones metalográficas.

Los resultados del análisis metalográfico muestran que la zona central (eje) de los lingotes experimentales es más densa y el grano de la estructura primaria es más pequeño. En los lingotes de control no existen grietas en la zona del eje central. La porosidad de la zona del eje central, en las probetas longitudinales de los lingotes de las coladas de control, es superior en un grado, y el diámetro máximo de los poros es tres veces mayor que en las coladas experimentales.

La diferencia en grados de las bandas de la segregación es muy grande. En las probetas experimentales se valoran en $0-1$, y en las de control en 0 $2,5^{\circ}$. La zona de superficie solidificada (zona de cristales basales) en los lingotes experimentales es dos veces superior y, lo más importante, la zona central es un $10 \%$ menor que en los lingotes de control.

La densidad de la estructura dendrítica se determina en las probetas longitudinales y transversales mediante un microanalizador marca Epikvant de la empresa Karl Zeiss. Las mediciones se realizan en secciones, ubicadas en la zona central del lingote y a la distancia de un cuarto de la longitud de la parte ancha de la superficie sobre el eje del lingote.

La dispersión de las dendritas se determina como la relación entre la cantidad de dendritas y la suma total de sus longitudes más la longitud de las distancias interdendríticas. El diámetro medio de las dendritas se determina por la relación entre la longitud de todas las dendritas y su cantidad. Los resultados de las determinaciones según los valores promedio de estas tres mediciones se incluyen en la tabla I.

Para determinar la influencia externa en el tamaño de la dendrita y la dispersión se toma la media de los valores obtenidos. El tamaño de la dendrita se determina según la ecuación:

$$
D=\left(X^{2}+y^{2}\right)^{1 / 2}
$$

donde

$X$ e $y=$ dimensiones_longitudinales y transversales de la dendrita, $\mu \mathrm{m}$.

La dispersión se determina mediante media aritmética.

La variación de la estructura de los lingotes ocasiona el aumento de sus propiedades mecánicas. 
TABLA I.- Influencia del campo eléctrico en los parámetros de la estructura dendrítica de los lingotes de acero TABLE I.- Influence of electrical field in the parameters of dendritic structure steel slabs

\begin{tabular}{|c|c|c|c|c|c|c|}
\hline \multirow{2}{*}{$\begin{array}{l}\text { Distancia desde } \\
\text { el borde estrecho } \\
\text { del lingote }\end{array}$} & \multicolumn{2}{|c|}{ Lingote experimental } & \multicolumn{2}{|c|}{ Lingote de control } & \multirow{2}{*}{$\begin{array}{l}\text { Disminución del } \\
\text { diámetro de la } \\
\text { dendrita, \% }\end{array}$} & \multirow{2}{*}{$\begin{array}{c}\text { Aumento de } \\
\text { dispersión de la } \\
\text { estructura del } \\
\text { lingote, \% }\end{array}$} \\
\hline & $\begin{array}{c}\text { Tamaño de } \\
\text { dendrita, } \mu \mathrm{m}\end{array}$ & $\begin{array}{c}\text { Dispersión, } \\
\mathrm{L} / \mu \mathrm{m}\end{array}$ & $\begin{array}{c}\text { Tamaño de } \\
\text { dendrita, } \mu \mathrm{m}\end{array}$ & $\begin{array}{c}\text { Dispersión, } \\
\mathrm{L} / \mu \mathrm{m}\end{array}$ & & \\
\hline & & $201 / 202$ & $345 /$ & $1,68 /$ & 26 & 84 \\
\hline $0,50 \mathrm{~L}$ & $377 / 286$ & $1,95 / 2,32$ & $426 / 354$ & $1,88 / 1,67$ & 14,6 & 20,2 \\
\hline
\end{tabular}

En el numerador se incluyen los valores de las muestras para los tamaños longitudinales de las probetas, en el denominador para los transversales.

Conjuntamente con esto, el análisis de la correlación de las dimensiones longitudinales y transversales de la dendrita en las probetas experimentales y de control (Tabla I) permite concluir que, en presencia del campo eléctrico, ocurre alguna variación de la textura, es decir el crecimiento de cristales en la dirección del campo eléctrico.

Anteriormente, en el microscopio electrónico se observaron fenómenos semejantes durante el crecimiento esferolítico del selenio en presencia de un campo eléctrico constante (9).

La investigación de la estructura fina del acero se realiza mediante filmación en un equipo de difracción de rayos $\mathrm{X}$. El cálculo de los parámetros de la estructura fina se realiza por el método de aproximación según las líneas (110), (200), (211) y (220). Se analiza el ensanchamiento de los máximos de la difracción para las líneas correspondientes $\left(\mathrm{Fe}_{\mathrm{k} \beta}=1,75653 \AA\right)$.
En la tabla II se incluyen las correlaciones de intensidad de las líneas del espectro de difracción que confirman la presencia de un alargamiento en la dirección (200) de las líneas de los cristales en los lingotes sometidos a influencias electrofísicas. Las determinaciones de la microdistorsión y de la zona de dispersión coherente permiten pronosticar las variaciones de las propiedades mecánicas del acero elaborado.

El aumento de la microdistorsión confirma el endurecimiento del metal y debe originar el aumento de las características de estabilidad (solidez) del lingote. La consolidación de los bloques (zonas de dispersión coherente) causa el ablandamiento del metal y un aumento de sus propiedades viscosas.

Los ensayos mecánicos de las probetas de acero fundido muestran que el límite de rotura del metal, sometido a influencias electrofísicas, es superior en un $11 \%$ al de las probetas de control.

TABLA II.- Resultados del análisis por difracción de rayos X de las probetas longitudinales (1-4) y transversales (5-8)

TABLE II.- Results of diffraction X-ray analysis of longitudinal (1-4) and transverse (5-8) test pieces

\begin{tabular}{|c|c|c|c|c|c|c|c|c|}
\hline \multirow{2}{*}{$\begin{array}{l}\text { Núm. de } \\
\text { probeta }\end{array}$} & \multirow{2}{*}{$\begin{array}{l}\text { Distancia desde } \\
\text { el borde estrecho } \\
\text { del lingote, } \mathrm{L}\end{array}$} & \multirow{2}{*}{ Lingote } & \multicolumn{4}{|c|}{ Intensidad de línea } & \multirow{2}{*}{$\begin{array}{l}\text { Micro- } \\
\text { distorsión }\end{array}$} & \multirow{2}{*}{$\begin{array}{c}\text { Tamaño de la } \\
\text { zona de dispersión } \\
\text { coherente } \mathrm{D}, \AA\end{array}$} \\
\hline & & & (110) & (200) & (211) & (220) & & \\
\hline 1 & 0,25 & $\mathrm{a}$ & 100 & 45 & 30 & 16 & 4,83 & 3.160 \\
\hline 2 & 0,25 & $\mathrm{~b}$ & 100 & 11 & 80 & 13 & 1,91 & 2.020 \\
\hline 3 & 0,50 & $\mathrm{a}$ & 100 & 30 & 36 & 16 & 6,35 & 2.540 \\
\hline 4 & 0,50 & b & 100 & 25 & 50 & 12 & 2,41 & 2.080 \\
\hline 5 & 0,25 & $\mathrm{a}$ & 100 & 40 & 43 & 10 & 9,51 & 3.025 \\
\hline 6 & 0,25 & b & 100 & 13 & 64 & 11 & 4,18 & 1.900 \\
\hline 7 & 0,50 & $\mathrm{a}$ & 100 & 45 & 44 & 15 & 7,03 & 2.870 \\
\hline 8 & 0,50 & $\mathrm{~b}$ & 100 & 30 & 50 & 18 & 2,31 & 2.120 \\
\hline \multicolumn{3}{|c|}{ Valores estándar } & 100 & 15 & 38 & 10 & - & - \\
\hline
\end{tabular}

a - Lingotes experimentales.

$\mathrm{b}$ - Lingotes de control. 


\section{CONCLUSIONES}

Los resultados obtenidos en ensayos de laboratorio con estaño fundido en crisol y a escala industrial con acero líquido vertido en moldes refrigerados, sometiendo estos metales a influencias electrofísicas, permiten establecer las siguientes conclusiones:

- El metal fundido durante la cristalización es un conductor heterogéneo. Por tanto, en él aparecen cargas eléctricas que interactúan desde un campo eléctrico externo de forma tal que el propio campo eléctrico del metal se reestructura. Esto debe ejercer influencia en los procesos de trasmisión de calor y de masa.

- Los ensayos de laboratorio muestran que durante la solidificación del metal en presencia de un campo eléctrico varía, tanto la temperatura de cristalización como el tiempo de solidificación.

- Los ensayos en una máquina de colada continua de palanquillas confirman la efectividad de utilización de las influencias electrofísicas en la cristalización del acero. Se elaboró la tecnología de obtención de lingotes de acero de forma continua con mejora de las propiedades mecánicas.

\section{REFERENCIAS}

(1) Vives, Ch. y Ricou, R. Met. Trans. B, 16B (2), 1985: 377 384.

(2) Sakane, J., LI, B.Q. y Evans, J.W. Met. Trans. B, 19B (2), 1988: 397-408.

(3) Barbier, J.N., Fautrelle, Y.R., Evans, J.W. y Cremer, P.J. Méch. Théorique Appliquée, (1), 1983: 533-539.

(4) Takeuchi, E., Miyoshino, I., Kawai, H., Tsunenari, K. y SAKANE, J. Proc. of the 6th Int. Iron and Steel Congress. ISIJ. Tokyo (Japón), 1990: 408-415.

(5) NAKATA, H. y ETAY, J. ISIJ Int., 32 (4), 1992: 521-528.

(6) SHKLYAR, V.S. Electronaya obrabotka metallov, (6), 1994: 35-37.

(7) Shklyar, V.S. Proc. of the Int. Symp. on Electromagnetic Processing of Materials, ISIJ. Nagoya (Japón), 1994: 572574.

(8) WINEGARD, W.C. An introduction to the solidification of metals. The'Institute of Metals. Londres, 1964.

(9) Alexandrov, V.D. y ShKlyar, V.S. Rev. Fizicheskoyjimii, (8), 1981: 2.093-2.094 